\title{
Erratum to: Topology Optimization of Compliant Mechanisms
}

\section{Erratum to:}

X. Zhang and B. Zhu, Topology Optimization of Compliant

Mechanisms, https://doi.org/10.1007/978-981-13-0432-3

In the original version of the book, the incorrect first author name "Xianming Zhang" has to be corrected to read as "Xianmin Zhang" in Frontmatter and Cover. The erratum book has been updated with the change. 